\title{
Comparative study of deterministic and stochastic pedestrian motions
}

\author{
Raj Kumar Pradhan ${ }^{1,2^{*}}$, Samir Shrestha ${ }^{2}$ and Sudarshan Tiwari ${ }^{3}$ \\ ${ }^{I}$ Khwopa College, Bhaktapur, Nepal. \\ ${ }^{2}$ Department of Mathematics, Kathmandu University, Nepal. \\ ${ }^{3}$ Department of Mathematics, University of Kaiserslautern, Germany.
}

Submitted: 10 March 2018; Revised: 28 August 2018; Accepted: 28 September 2018

\begin{abstract}
The various collective phenomena observed in pedestrian crowds-flow have attracted interest of the rapidly increasing number of scientists, planners and designers. The implementation of a traffic policy without pedestrian analysis might lead to a costly trial and error due to implementation cost. The risk of crowd panic during an emergency or disaster can be minimised by developing a mathematical model mimicking the evacuation strategies. In this study, we estimated the evacuation time for deterministic and stochastic pedestrians flows, which are the system of ordinary differential equations (ODEs), using microscopic model based on social force model. We used stochastic differential equations (SDEs) to model the uncertainty nature of pedestrian motion. Numerical simulations were carried out for one dimensional pedestrians motion and numerical results were compared for various parameters in the models.
\end{abstract}

Keywords: Deterministic and stochastic pedestrian flows, microscopic model, pedestrian flow, social force.

\section{INTRODUCTION}

Pedestrian flow models have many applications. It is important for designing and planning pedestrian areas, public buildings, religious places, bus stations, theaters, stadiums etc., in terms of the capacity and also with respect to the issues such as safety and evacuation. Foot traffic is another area of application of pedestrian flow models. The exact knowledge of pedestrian dynamics plays an essential role in planning major events, where a high density of pedestrians are expected. The organisers require correct information to develop and test the management strategies on what areas are likely to be congested, before implementation. Experiments are the best method to identify the behaviour of pedestrians in various situations, but performing these in reality is difficult, expensive, time consuming, and impractical. Therefore, experimentally simulating pedestrian flow in stress situations is not possible. In these situations, pedestrian models are used.

Pedestrian dynamic simulations are important tools to support efficient crowd control. Many different simulation models exist in this field. These models can be distinguished into three different types according to their spatial resolution as models from the microscopic, mesoscopic and macroscopic levels (Teknomo \& Gerilla, 2008; Biedermann \& Borrmann, 2016).

Microscopic models provide the highest spatial resolution for the cost of highest computational effort compared to simulation models from other spatial scales. These models simulate individual pedestrians on a continuous simulation scenario. This means that their spatial resolution is unrestricted. The social force model developed by Helbing et al. (2002) is a well-known example of a microscopic model. Macroscopic models analyse the pedestrians in groups and crowds where the state of the system is generally described by number density, average velocity, etc. Mesoscopic models usually describe the pedestrian entities at a higher a

\footnotetext{
*Corresponding author (rajpradhan63@gmail.com; (10) https://orcid.org/0000-0002-6196-8264)
} 
level of detail, but their behaviour and interactions are described at a lower level of detail. These models do not distinguish each pedestrian individually and report the pedestrian characteristics in aggregate terms. Macroscopic and mesoscopic pedestrian models are generally appropriate to study huge crowd dynamics in which the analysis of detailed interaction among the pedestrians is computationally expensive. In this paper, we have considered a maximum of 230 pedestrians and hence the microscopic model is found to be appropriate for the study. There are narrow pathways/walkways in different pedestrian areas such as footpaths/sidewalks along the roadways and banks of rivers, narrow walkways in dense residential areas, narrow corridors in civil malls and hospitals, lanes in front of temples and public service offices, narrow bridges over rivers where just one or two persons pass through at a time. Pedestrian motion in these situations seem to be one-dimensional. In this paper, we present the motion of pedestrians in one dimension.

Previous researchers (Teknomo, 2002; Daamen, 2004; Johansson, 2013; Etikyala et al., 2014) have studied the microscopic modelling of deterministic pedestrian flow on the basis of social force model. Hence we have presented the microscopic pedestrian flow on the basis of social force model by incorporating the stochastic nature in the deterministic models in our onedimensional study of pedestrian flow. The numerical simulations and graphical visualisations were performed using the computer software MATLAB 9.1/R2016b.

\section{MATHEMATICAL MODELLING}

We present the microscopic models of pedestrian flow in the form of a system of differential equations. To study the unpredictable nature of pedestrian decision making, we use a system of stochastic differential equations. We consider a domain $\Omega \subseteq R^{d}, d=1,2$ of $N$ number of pedestrians in which the position of $i^{\text {th }}$ pedestrian at time $t$ is $X_{i}=X_{i}(t), i=1,2,3, \cdots, N$. Each pedestrian has its own intended velocity to reach the destination. In the study, the nature of the movement of the pedestrians while interacting with neighbours is presented. The behaviour of the pedestrians is mathematically presented in the following subsection.

\section{Equations of pedestrians flow}

We have considered a microscopic social force model for pedestrian flow, which includes an optimal path computation. It is developed by coupling the classical social force models (Helbing, 2001; Helbing et al.,
2002) with an optimal path computation as in the Hughes (2002) approach for pedestrian flow. We have discussed the interacting particle system with locations at $X_{i} \in \Omega \subseteq R^{d}, i=1,2, \cdots, N$ and the velocity is $v_{i} \in R^{d}$. The equations of the pedestrian flow (Etikyala et al., 2014) are,

$\left.\begin{array}{l}d X_{i}=v_{i} d t \\ d v_{i}=1 / \tau\left(U_{i}\left(\rho_{i}\right) e-v_{i}\right) d t+\sum_{i \neq j} F_{i j}\left(X_{i}-X_{j}, v_{i}-v_{j}\right) d t+\sigma d W_{i}(t)\end{array}\right\}$

where, $\rho=\rho(X)=\frac{1}{N_{\max }^{R}} \sum_{j,\left\|X-X_{j}\right\|<R} 1$ and $\rho_{i}=\rho\left(X_{i}\right)$.

Here, $\rho(X)$ is the number density of the pedestrians, $N_{\max }^{R}$ denotes the maximum number of pedestrians in a ball of radius $R$ and $\tau$ is the reaction time describing how fast the pedestrians can correct their current velocity $v_{i}$ to the desired velocity $U_{i} e$. The desired speed is $U_{i}:[0,1] \rightarrow\left[0, U_{\max }\right]$ where $U_{\max }$ is the maximum comfort speed of the pedestrians given by the Greenshield's speed density relation (Greenshields, 1935).

$U(\rho)=U_{\max }\left(1-\frac{\rho}{\rho_{\max }}\right)$

The direction of the destination of the $i^{\text {th }}$ pedestrian is given by $e$ which is defined as,

$e=-\frac{\nabla \phi(X)}{\|\nabla \phi(X)\|}$

where, $\phi(X)$ is the solution of the Eikonal equation (Etikyala et al., 2014 ).

The interaction force is described by,

$F_{i j}(X, v)=F^{(1)}(X)+F^{(2)}(X, v)$

The first term $F^{(1)}(X)$ on the right hand side indicates the elastic force and the second term $F^{(2)}(X, v)$ represents the dissipative force. The elastic force $F^{(1)}(X)$ is defined by,

$F^{(1)}(X)=F_{\text {elas }}(X)=-\nabla_{X} \psi_{1}(\|X\|)$

where, $\psi_{1}$ is the interaction potential given by,

$\psi_{1}(X)=k_{n}\left(2 R^{2}-\|X\|\left(2 R-\frac{\|X\|}{2}\right)\right) H(2 R-\|X\|)$

where, $H$ is the Heaviside function. 
After some mathematical simplification equation (5) can be written as,

$$
F^{(1)}(X)=F_{\text {elas }}(X)=k_{n} n(X)(2 R-\|X\|) H(2 R-\|X\|)
$$

where, $n(X)=\left(n_{x}, n_{y}\right)=\frac{X}{\|X\|}$ is the normal unit vector. This force is complemented by a dissipative force (Helbing et al., 2002; Etikyala et al., 2014) $F^{(2)}(X, v)$. The dissipative force is given by,

$$
F^{(2)}(X, v)=F_{d i s s}^{(2)}=\left(F_{n}^{(2)}+F_{t}^{(2)}\right) H(2 R-\|X\|)
$$

Here, the normal dissipative force is given by $F_{n}^{(2)}(X, v)=-\gamma_{n}<v, n>n$ where, $<\cdot, \cdot>$ denotes the scalar product. The tangential dissipative force is $F_{t}^{(2)}(X, v)=-\gamma_{t} v^{t}=-\gamma_{t}<v, n^{\perp}>n^{\perp}$

where, $v^{t}=v-<v, n>n$ is the tangential component of the relative velocity $v$ pointing into the direction of $n^{\perp}$, which is normal to $n . R$ denotes the radius of comfort distance of the pedestrians, $k_{n}$ is the interaction constant and $\gamma_{n}$ and $\gamma_{t}$ are suitable positive friction constants. In one-dimensional pedestrian motion, the tangential force is zero. The last term of equation (1) that involves the Brownian motion or Wiener process $W_{i}$ (Kloeden \& Eckhard, 1999), is the diffusion term which describes the unpredictable nature of the $i^{t h}$ pedestrian and $\sigma>0$ is the strength of randomness.

\section{RESULTS AND DISCUSSION}

In this section, numerical experiments on microscopic pedestrian flow are presented. We compute and compare the evacuation time of the pedestrians based on the microscopic equation (1). We also analyse the evacuation time of the pedestrians for various parameter values seen in the flow model. The pedestrian flow models considering one-dimensional narrow walkway domain $D=[0, L]$ were investigated where only one pedestrian can walk along the walkway at a time. $N$ number of pedestrians located uniformly in the domain were considered. Each pedestrian has its own initial position, initial velocity and a predetermined destination. The interaction force $F_{i j}$ between the $i^{\text {th }}$ and $j^{\text {th }}$ pedestrian takes the form,

$$
F_{i j}^{1}= \begin{cases}-k_{n}\left(2 R-d_{i j}\right), & \text { if } d_{i j}<2 R \\ 0, & \text { Otherwise }\end{cases}
$$

where, $d_{i j}=\left|X_{i}-X_{j}\right|$ is the distance between $i^{\text {th }}$ and $j^{\text {th }}$ pedestrian, $R$ is the comfort radius of a pedestrian so that $2 R$ is the minimum distance between the $i^{\text {th }}$ and $j^{\text {th }}$ pedestrians at which they feel comfortable as shown in Figure 1. If $d_{i j}<2 R$, then pedestrians start to push away each other by a force $F_{i j}^{1}$. We have considered the pedestrians as having almost equal body dimensions with a comfort radius $R$. A forward force drives the pedestrians in the direction of their destination when there are no pedestrians in their comfort zone. The motion of each pedestrian is affected by the pedestrians just in front of his/her position in the direction of the destination. Therefore, in the computational domain, we count the number of pedestrians $N_{c}$ in a cell of length 5 $\mathrm{m}$ in front of each pedestrian in the direction of his/her destination.

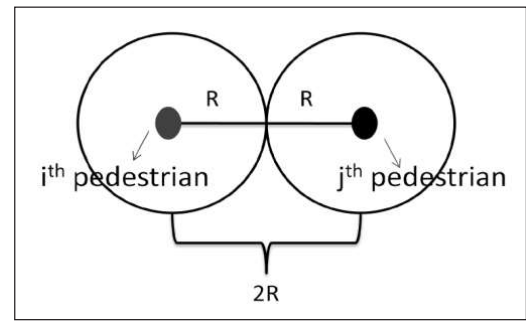

Figure 1: Comfort zone of two pedestrians

We define $N_{\max }$ as the maximum number of pedestrians in each cell. The number density $\rho$ of the pedestrians is calculated by using the formula,

$\rho=\frac{N_{c}}{N_{\max }}$

The system of differential equations (1) is solved numerically by using the Euler-Maruyama scheme. Euler-Maruyama scheme is an explicit scheme, which is stable for a small time step and mostly used to solve stochastic differential equations (Kloeden \& Eckhard, 1999) numerically. The system of differential equation models either deterministic or stochastic nature of the pedestrian flow when $\sigma=0$ or $\sigma \neq 0$, respectively.

The numerical scheme for the pedestrian motion is,

$$
\begin{aligned}
v_{i}(t+d t)= & v_{i}(t)+\frac{1}{\tau}\left(U_{i}\left(\rho_{i}(t)\right) e-v_{i}(t)\right) d t+\sum_{i \neq j}\left(F_{i j}^{(1)}(t)\right. \\
& \left.+F_{i j}^{(2)}(t)\right) d t+\sigma \sqrt{d t} \xi_{i} \quad \xi_{i} \sim N(0,1) \quad \ldots(11)
\end{aligned}
$$

$X_{i}(t+d t)=X_{i}(t)+v_{i}(t) d t$ 
where, $e$ gives the direction of the pedestrians which will be +1 or -1 according to the direction of the destination. In our numerical experiment, we have used the value of parameters as $L=200 \mathrm{~m}$ and $\tau=0.5 \mathrm{~s}$ fixed for all the experiments and the evacuation time is calculated and compared by varying the values of other parameters such as $N, R, k_{n}, \gamma_{n}, U_{\max }$ and $\sigma$. The physical units of the parameters $k_{n}, \gamma_{n}$ and $\sigma^{2}$ are $\mathrm{kg} / \mathrm{s}^{2}, \mathrm{~kg} / \mathrm{s}$, and $\mathrm{m} / \mathrm{s}$, respectively. We solve the above equations to compute the evacuation time of the pedestrians through three different exit locations.

\section{Numerical results for a single exit}

In this section, we present a numerical experiment for the pedestrians in the domain exiting through a single exit at the right extremity and estimate the average evacuation time for the different number of pedestrians in a narrow domain of length $L=200 \mathrm{~m}$ keeping the parameters $\sigma=0.5, \quad k_{n}=2, \quad \gamma_{n}=0.2$, and $R=1 \mathrm{~m}$ constant, and compare the results for the different models. The experimental setup for the one-dimensional walkway is shown in Figure 2.

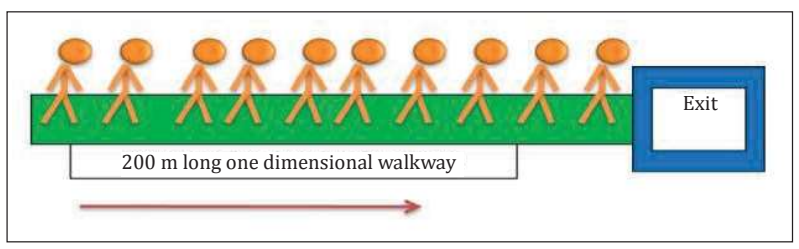

Figure 2: Experimental setup for pedestrian flow through single exit at right extremity

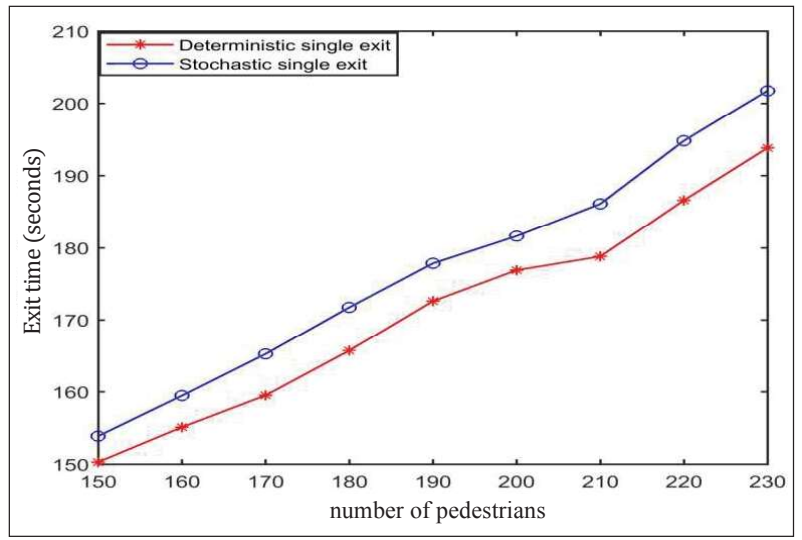

Figure 3: Relationship between average evacuation time and number of pedestrians through a single exit
Numerical results for the evacuation time and the comparisons for the different number of pedestrians with a maximum speed of $U_{\max }=2 \mathrm{~m} / \mathrm{s}$ through a single exit are presented in Figure 3.

Numerical results for the evacuation time and the comparisons for 230 pedestrians for different maximum speeds $U_{\max }$ in a narrow domain $L=200 \mathrm{~m}$ through a single exit for the different models are presented in Figure 4.

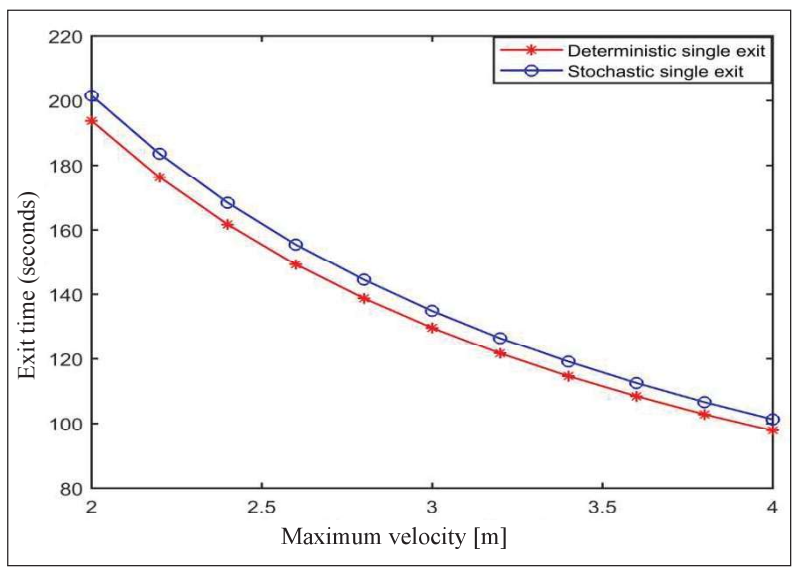

Figure 4: Relationship of evacuation time and maximum speed of pedestrians through a single exit

\section{Numerical results for two exits at the extremities}

In this section, we present the numerical experiment for the pedestrians in a narrow domain of length $L=200 \mathrm{~m}$ keeping the parameters $\sigma=0.5, k_{n}=2, \gamma_{n}=0.2$ and $R=1 \mathrm{~m}$ constant. Pedestrians exit through two exits at the extremities. Pedestrians near to the right exit move through the right exit and those who are near to the left exit move through the left exit. The experimental setup is shown in Figure 5.

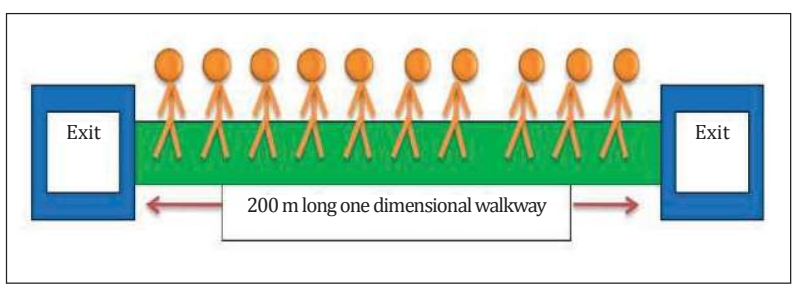

Figure 5: Experimental setup for pedestrian flow through two exits at the extremities 
Numerical results for the evacuation time and the comparisons for different number of pedestrians with a maximum speed of $U_{\max }=2 \mathrm{~m} / \mathrm{s}$ through two exits are presented in Figure 6.

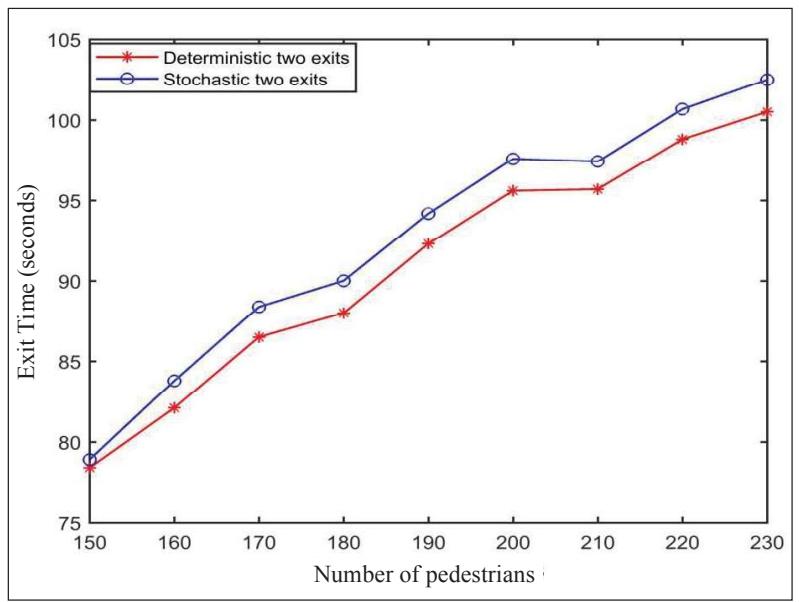

Figure 6: Relationship of average evacuation time and number of pedestrians through two exits at the extremities

The numerical results for evacuation time and the comparisons for 230 pedestrians for different maximum speeds $U_{\max }$ in a narrow domain of length $L=200 \mathrm{~m}$ through two exits at the extremities for the different models are presented in Figure 7.

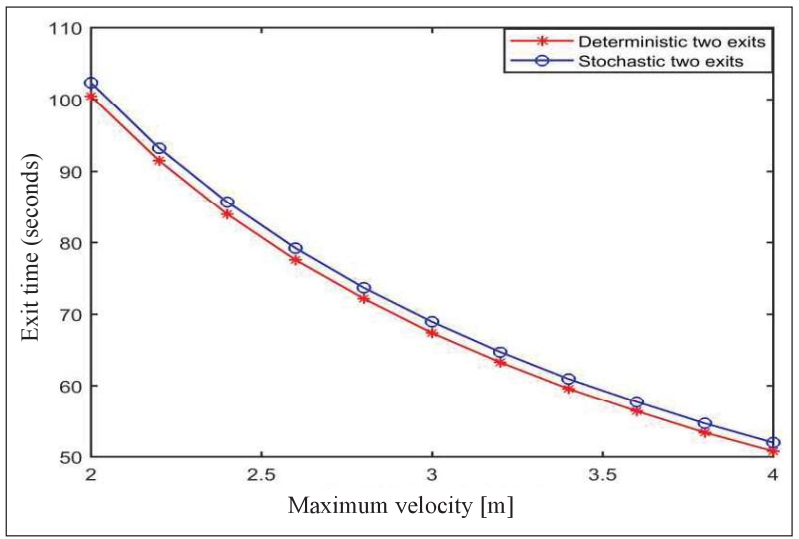

Figure 7: Relationship between evacuation time and maximum speed of pedestrians through two exits at the extremities

\section{Numerical results for a single middle exit}

Numerical experiments for the pedestrians in a narrow domain of length $L=200 \mathrm{~m}$ are presented keeping the parameters $\sigma=0.5, k_{n}=2, \gamma_{n}=0.2$, and $R=1 \mathrm{~m}$ constant. Pedestrians are evacuated through a single middle exit. The experimental setup is shown in Figure 8.

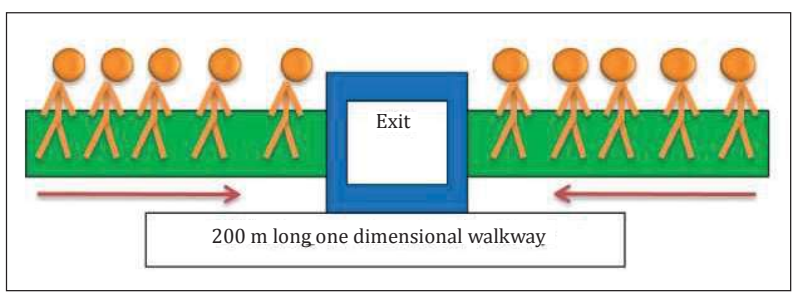

Figure 8: Experimental setup for pedestrian flow through a single middle exit

Numerical results for the evacuation time and the comparisons for different number of pedestrians with a maximum speed of $U_{\max }=2 \mathrm{~m} / \mathrm{s}$ through a single middle exit are presented in Figure 9.

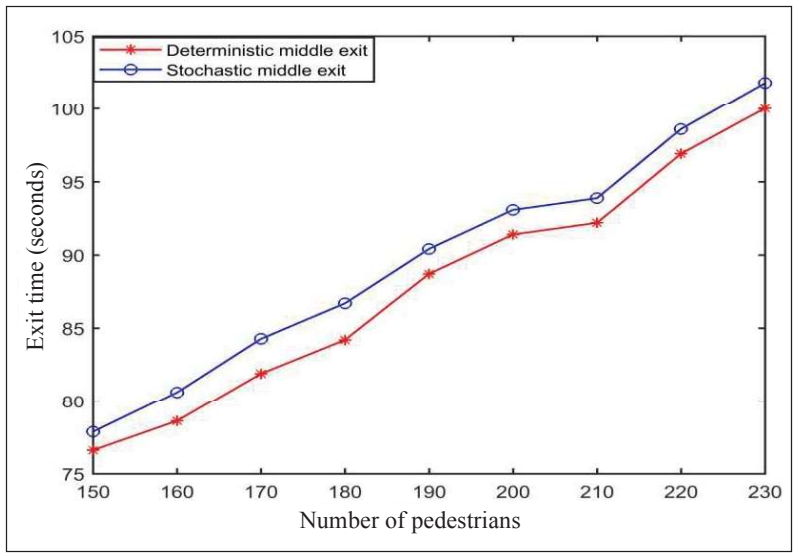

Figure 9: Relationship of average evacuation time and number of pedestrians through a single middle exit

The numerical results for evacuation time and the comparison for 230 pedestrians for different maximum speeds $U_{\max }$ in a narrow domain $L=200 \mathrm{~m}$ through a single middle exit, for different models are presented in Figure 10. 


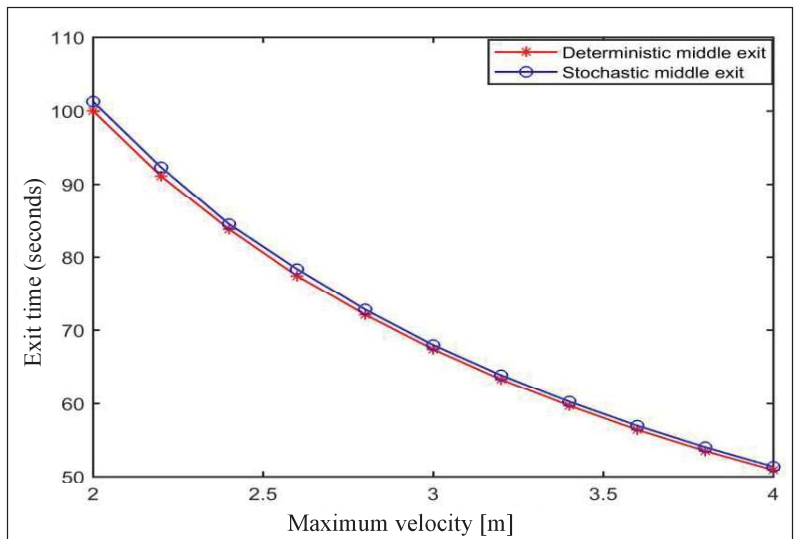

Figure 10: Relationship of evacuation time and maximum speed of the pedestrians through a single middle exit

Figures 3, 4, 6, 7, 9 and 10 show that the evacuation time for the deterministic pedestrians is smaller than that of the stochastic pedestrians, which implies that the deterministic pedestrians can be evacuated faster than the stochastic pedestrians.

\section{Comparison of evacuation time through different exits}

In this section, we present a comparison of the average evacuation time for deterministic and stochastic pedestrian flow in a single lane through different exits. Through the numerical experiment, it is observed that the average evacuation time for both deterministic and stochastic pedestrians through a single middle exit is shorter than the other two types of exits. We

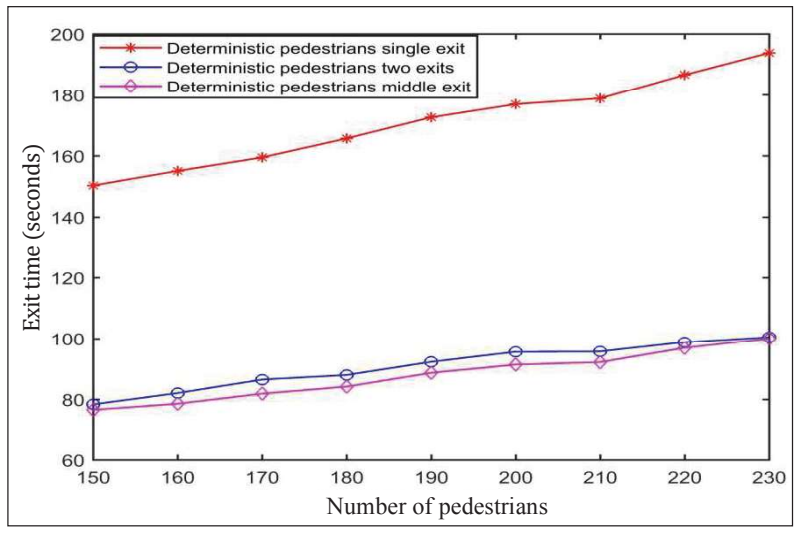

Figure 11: Comparison of evacuation time of deterministic pedestrians through different exits have presented the estimated evacuation time and the comparisons with different models in Figures 11 and 12.

Figure 11 depicts the relationship between average evacuation time and the number of pedestrians for different exits. It was observed that the average evacuation time difference of the deterministic pedestrians through a single exit and two exits is $80.16 \mathrm{~s}$ whereas the difference between the single exit and middle exit is $83.20 \mathrm{~s}$. The average time difference of the deterministic pedestrians through two exits and the middle exit is $3.04 \mathrm{~s}$ which is due to the density of the pedestrians ahead of them in the moving direction.

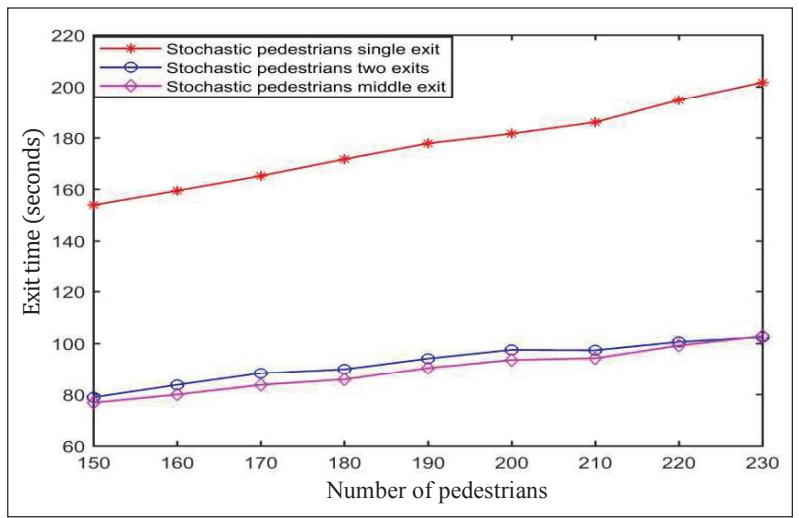

Figure 12: Comparison of evacuation time of stochastic pedestrians for different exits

The same phenomenon as for the deterministic pedestrians is observed for the stochastic pedestrians. The average evacuation time difference of the stochastic pedestrians through single exit and two exits is $84.33 \mathrm{~s}$, whereas the difference between the single exit and middle exit is $87.26 \mathrm{~s}$. The average time differences of the stochastic pedestrians through two exits and the middle exit is $3.04 \mathrm{~s}$.

From the above discussion, we conclude that the pedestrians can be evacuated faster through the middle exit than the other two types of exits.

\section{Comparison of evacuation time for different values of parameters}

The comparison of average evacuation time for different parameters $\gamma_{n}, k_{n}, R$ and $\sigma$ in a single lane $L=200 \mathrm{~m}$ for different number of pedestrians with a maximum speed of $U_{\max }=2 \mathrm{~m} / \mathrm{s}$ through a single exit is presented in this section. 


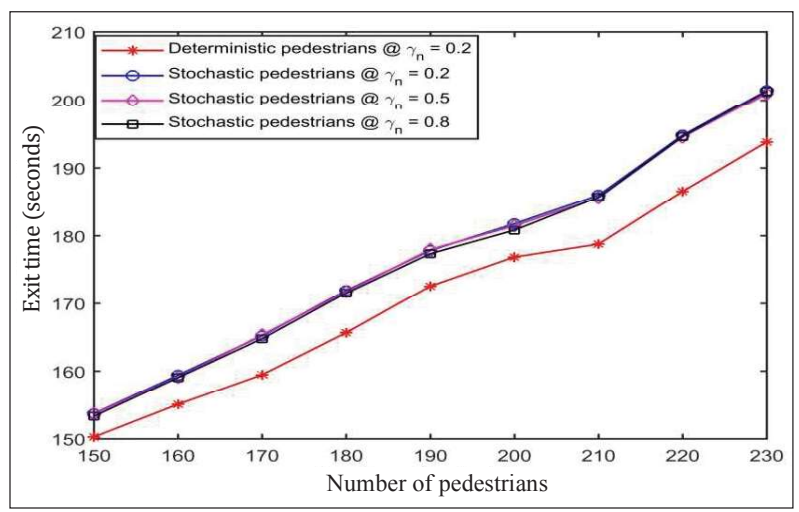

Figure 13: Comparison of evacuation time of the pedestrians by varying dissipative coefficient $\gamma_{n}$

From Figure 13 it can be observed that when the value of dissipative coefficient increases the average evacuation time decreases.

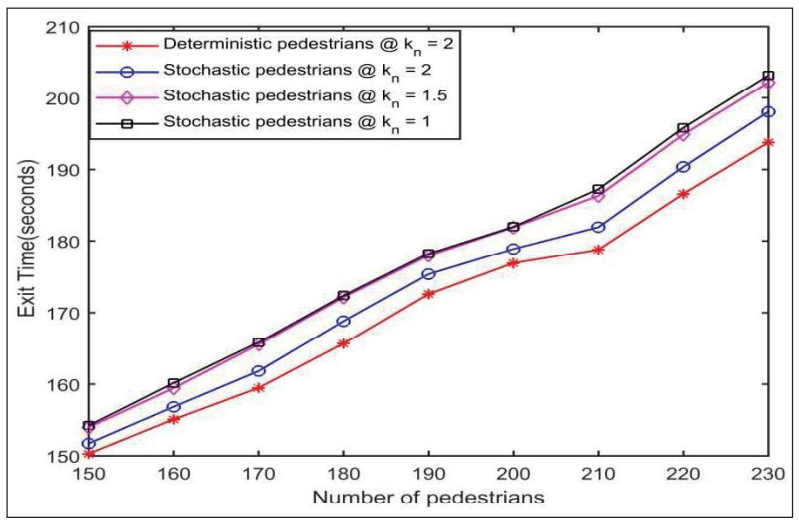

Figure 14: Comparison of evacuation time of pedestrians varying elastic coefficient $k_{n}$.

Figure 14 show the sensibility analysis of the different number of the pedestrians through a single exit with a maximum speed of $U_{\max }=2 \mathrm{~m} / \mathrm{s}$ for different values of the elastic coefficient $k_{n}$. It was observed that when the value of $k_{n}$ increases, the evacuation time decreases.

Figure 15 shows that when the comfort distance $R$ of the pedestrians increases, the evacuation time also increases. This is due to the fact that if the pedestrians have a greater comfort distance, there is more interaction between the pedestrians. Pedestrians push adjacent pedestrians far away so that it takes more time to evacuate them.

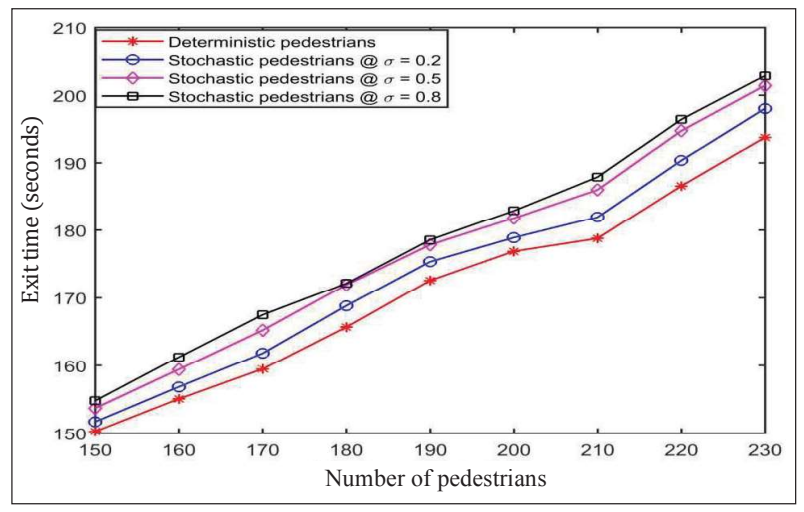

Figure 15: Comparison of evacuation time of the interacting pedestrians by varying the comfort radius $R$

The numerical results for the comparisons of evacuation time of the deterministic and stochastic pedestrians are presented in Figure 16.

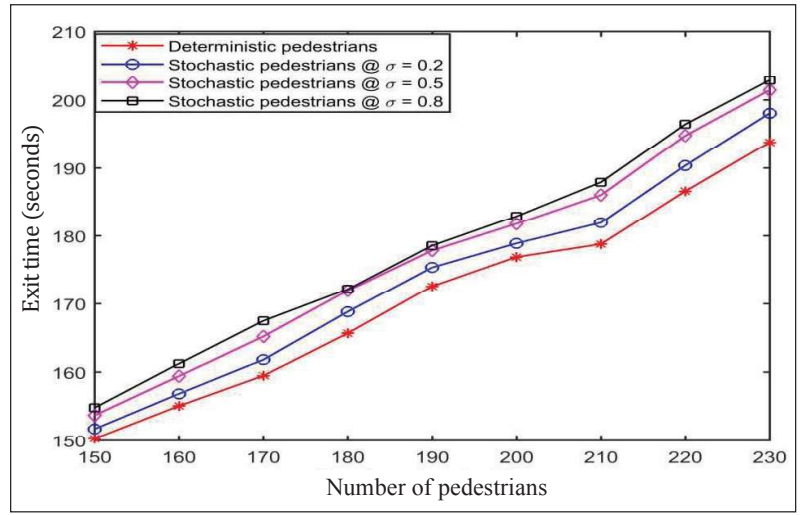

Figure 16: Comparison of the evacuation time of the pedestrians by varying diffusion coefficient $\sigma$.

Figure 16 shows that when the strength of $\sigma$ increases, the evacuation time also increases. It is due to the fact that the larger the strength of randomness, more the uncertainty behaviour of the pedestrians, resulting in a long time for the evacuation of the pedestrians.

\section{CONCLUSIONS}

The pedestrian flow models with and without stochastic behaviors are presented in this paper. The evacuation time through different exit locations in one-dimensional pathway is numerically estimated. The study concludes that the deterministic pedestrians can be evacuated faster 
than the pedestrians walking randomly. It is also observed that pedestrians can be evacuated faster by increasing the comfort velocity of the pedestrians. Pedestrians can be evacuated faster in a single lane with a middle exit. Smaller comfort radius of the pedestrians lowers the evacuation time, whereas strength of randomness $\sigma$ increases the evacuation time. In this study, the parameters values are taken randomly.

\section{Acknowledgement}

The authors acknowledge the University Grants Commission (UGC), Nepal for providing research support for this study under the M Phil Research Support (Award No. MPhRS-73/74-S\&T-03).

\section{REFERENCES}

Biedermann D.H. \& Borrmann A. (2016). A generic and hybrid approach for pedestrian dynamics to couple cellular automata with network flow models. Proceedings of the $8^{\text {th }}$ International Conference on Pedestrian and Evacuation Dynamics (PED 2016) (eds. W. Song, J. Ma \& L. Fu), volume 1, Hefei, China, 12-17 October, pp. 236-242.

DOI: https://doi.org/10.17815/CD.2016.11

Daamen W. (2004). Modelling passenger flows in public transport facilities. PhD thesis, Delft University of Technology, Faculty of Civil Engineering and Geosciences, The Netherlands.

Etikyala R., Göttlich S., Klar A. \& Tiwari S. (2014). Particle methods for pedestrian flow models: from microscopic to non-local continuum models. Mathematical Methods and Models in Applied Sciences 24(12): 2503-2523. DOI: https://doi.org/10.1142/S0218202514500274

Greenshields B.D. (1935). A study of highway capacity. Proceedings of the Highway Research Board, pp. 448-477. Highway Research Board, Washington DC, USA.

Helbing D. (2001). Traffic related self-driven many particles systems. Review of Modern Physics 73(4): 1067-1141. DOI: https://doi.org/10.1103/RevModPhys.73.1067

Helbing D., Farkas I.J., Molnar P. \& Vicsek T. (2002). Simulation of pedestrian crowds in normal and evacuation situations. Pedestrian and Evacuation Dynamics (eds. M. Schreckenberg \& S.D. Sharma), pp. 21-58. SpringerVerlag Berlin, Germany.

Hughes R.L. (2002). A continuum theory for the flow of pedestrians. Transportation Research Part B: Methodological 36(6): 507-535. DOI: https://doi.org/10.1016/S0191-2615(01)00015-7

Johansson F. (2013). Microscopic modeling and simulation of pedestrian traffic. PhD. thesis, Linkoping University, Sweden.

Kloeden P.E. \& Eckhard P. (1995). Numerical Solution of Stochastic Differential Equations. volume 23, SpringerVerlag, Berlin, Heidelberg, Germany.

Teknomo K. (2002). Microscopic pedestrian flow characteristics: development of an image processing data collection and simulation model. PhD thesis, Tohoku University, Japan.

Teknomo K. \& Gerilla G.P. (2008). Mesoscopic multi-agent pedestrian simulation. Transportation Research Trends (eds. P.O. Inweldi), pp. 323-336. Nova Science Publishers, Inc., New York, USA. 